\title{
RESPONSABILIDADE SOCIOAMBIENTAL DAS EMPRESAS BRASILEIRAS DE ENERGIA ELÉTRICA SOCIAL AND ENVIRONMENTAL RESPONSIBILITY OF BRAZILIAN ELECTRICITY COMPANIES
}

Vera Lúcia Cruz ${ }^{1}$

\section{RESUMO}

O estudo objetivou investigar as informações sociais, ambientais e econômicas que são divulgadas pelos grupos brasileiros de distribuição de energia elétrica. Nesta pesquisa realizou-se uma abordagem qualitativa, a qual foi delineada por uma pesquisa documental por meio de um levantamento das informações divulgas pelas empresas de energia elétrica do Brasil, relativos ao exercício findo em 31/12/2017. Foi usado como base, as informações sociais, ambientais e econômicas apontadas no estudo de Siksnelyte e Stjepcevic (2017), buscando alinhar as ações das empresas brasileiras com o padrão das exigências internacionais. A pesquisa foi realizada com 33 distribuidoras de energia, que juntas, formam 14 grupos de empresas controladoras. Os principais resultados em relação ao estudo de Siksnelyte e Stjepcevic (2017) demonstram que duas empresas apresentaram percentual abaixo dos $60 \%$ dos itens abordados e que nos temas relacionados a responsabilidade ambiental, as empresas apresentaram uma média geral de $77 \%$, mostrando assim, a necessidade de avanço. Dessa forma, pôde-se concluir que as empresas estão gerando informações socioambiental, no entanto, assim como os estudos de Gonzaga et al., (2012) e Siqueira e Fernandes (2010), foi constatado a necessidade de melhorias nas ações realizadas pelas empresas de energia, tendo em vista o impacto que a atividade causa na natureza.

Palavras chave: Setor elétrico. Responsabilidade social. Responsabilidade ambiental. Sustentabilidade econômica.

1 Doutora em Administração pela Universidade Potiguar - UnP. Mestre em Ciências Contábeis pela Universidade Federal de Pernambuco - UFPE. Docente da Universidade Federal da Paraíba. E-mail: veralc22@hotmail.com

2 Especialista em Auditoria e Controladoria pelo Centro Universitário de João Pessoa - UNIPÊ. E-mail: arthur_machadp1-1@ hotmail.com

3 Especialista em Auditoria e Controladoria pelo Centro Universitário de João Pessoa - UNIPÊ. E-mail: dsfalcao991@ gmail.com

4 Doutor em Engenharia de Sistemas e Computação pela Universidade Federal do Rio de Janeiro - UFRJ. Docente do Programa de Pós-Graduação em Administração da Universidade Potiguar - UnP. E-mail: r.leone@uol.com.br 


\section{ABSTRACT}

The study aimed to investigate the social, environmental and economic information that are disclosed by the Brazilian electricity distribution groups. In this research a qualitative approach was carried out, which was delineated by a documentary research through a survey of the information disclosed by the Brazilian electric energy companies for the year ended 12/31/2017. The social, environmental and economic information pointed out in the study of Siksnelyte and Stjepcevic (2017) was used as a basis, seeking to align the actions of Brazilian companies with the standard of international requirements. The survey was conducted with 33 energy distributors, which together form 14 groups of controlling companies. The main results in relation to the study by Siksnelyte and Stjepcevic (2017) show that two companies presented a percentage below $60 \%$ of the items dealt with and that in the themes related to environmental responsibility, companies presented a general average of $77 \%$, thus showing the need for advancement. Thus, it could be concluded that companies are generating socio-environmental information, however, as well as studies by Gonzaga et al., (2012) and Siqueira and Fernandes (2010), it was verified the need for improvements in the actions carried out by energy companies, considering the impact that the activity has on nature.

Key-words: Electric sector. Social responsability. Environmental responsibility. Economic sustainability.

\section{INTRODUÇÃO}

A maneira pela qual as empresas interagem com a sociedade mudou ao longo do tempo e o conceito de Responsabilidade Social Empresarial (RSE) ganhou reputação, assim, as empresas têm assumido a responsabilidade por seus impactos na sociedade e no ambiente (SIDHOUM; SERRA, 2017). Aos poucos, pode ser observado que a RSE vem se tornando um fator de competitividade para os negócios e um diferencial para trazer novos investidores (CAMILO, et al., 2015).

Dessa forma, empresas que competem globalmente estão sendo direcionadas ao comprometimento de informações sobre o desempenho no âmbito da sustentabilidade empresarial das iniciativas operacionais (LABUSCHAGNE; BRENT; VAN ERCK, 2005). Corroborando Siksnelyte e Stjepcevic (2017) apontam que um número crescente de organizações estão se tornando cada vez mais ativas nas abordagens de problemas sociais. Entre essas empresas, encontram-se as empresas de energia elétrica cujo produto costuma ser um fator essencial na sociedade, além de oferecer maior bem-estar à população, sua utilização propicia o aumento da capacidade de produção de bens e serviços nos mais diversos setores da economia (FINKLER, et al., 2016).

Apesar do Brasil apresentar uma matriz energética considerada limpa, majoritariamente composta por fontes de energias renováveis que não causam poluição pela emissão de substância (FONTES, 2018), cada fonte, seja hidráulica, biomassa, eólica, solar ou nuclear apresentam um conjunto de impactos ambientais negativos. Dessa forma, os impactos ambientais negativos dessas empresas, provenientes do uso ou geração da energia elétrica afetam diretamente a comunidade, fazendo com que haja a necessidade que tratem de forma simultânea o conjunto de ações sociais e ambientais. E esses impactos, exigem das empresas de energia elétrica o planejamento, controle e o cumprimento de obrigações legais para a realização de suas atividades (BRAGA; FERREIRA, 2015). Aliado ao impacto que as empresas causam no meio ambiente, tanto em países desenvolvidos como os que estão 
em desenvolvimento têm-se o aumento da demanda por energia, assim como existe uma crescente pressão sobre a questão da mudança climática, que está fortemente relacionada com a eficiência de uso dos recursos energéticos (SIKSNELYTE; STJEPCEVIC, 2017).

Além disso, aumentou consideravelmente a preocupação das empresas com a Responsabilidade Social Corporativa (RSC) e o Desenvolvimento Sustentável. Isso porque novos públicos têm procurado informações além das financeiras que passam a ser usadas para fins de investimento (GODÓI-DESOUSA et al., 2009). Corroborando Lozano e Reid (2018) apontaram que o sistema global de energia representa um desafio crucial para o conceito de sustentabilidade e vice-versa. Nesse mesmo estudo, os autores apontam que os modelos tradicionais de geração de empresas de energia elétrica da Europa estão sendo cada vez mais desafiados pela sustentabilidade.

Dentro dessa perspectiva a Agência Nacional de Energia Elétrica - ANEEL (2017) aponta que a sustentabilidade vem se tornando meta consensual, expressa formalmente em termos globais. São exemplos os objetivos do milênio e objetivos de desenvolvimento sustentável (ODS), apontando para conquistas tecnológicas e mudanças comportamentais. Assim, o setor elétrico busca equacionar a necessidade do atendimento da demanda por energia elétrica e a complexidade ambiental do país, buscando aproveitamentos energéticos compatíveis com a sua realidade (CONFEDERAÇÃO NACIONAL DA INDÚSTRIA, 2017), tendo em vista que a dependência esmagadora da moderna sociedade baseada em fontes não renováveis de produção de energia baseadas em fósseis representa um grande desafio para a sustentabilidade (LOZANO; REID, 2018).

Porém, Maçambanni et al., (2013) buscaram identificar o nível de informação socioambiental fornecido pelas empresas de energia do setor elétrico listadas na B3 e verificaram que apenas 21 empresas apresentaram um nível de disclosure socioambiental próximo ao exigido. Além disso, a publicação dos indicadores sociais e ambientais mostrou um nível de evidenciação ainda muito aquém do esperado.

Destarte, evidencia-se a necessidade de responsabilidade de ações sociais das empresas de energia, assim, este estudo teve como base o artigo de Responsabilidade Social das Empresas no Setor de Energia das autoras Siksnelyte e Stjepcevic (2017) onde afirmam que o setor da indústria de energia é uma parte significativa da sociedade e deve ter um papel de portador do bem-estar. As autoras apresentam três pilares do desenvolvimento sustentável nesse segmento: Responsabilidade Social, Responsabilidade Ambiental e Responsabilidade Econômica, e, em cada uma dessas bases, colocam as questões mais importantes que precisam ser executadas, e consequentemente, evidenciadas nos relatórios gerados pelas empresas do setor de energia elétrica. Siksnelyte e Stjepcevic (2017) complementam afirmando que a responsabilidade econômica e socioambientais pode ajudar a gerenciar a escassez de recursos provocadas por sua própria atividade através de uma melhor integração de metas.

Diante do exposto, este trabalho buscou responder a seguinte questão: Qual as informações divulgadas sobre responsabilidade social, ambiental e econômica dos grupos brasileiros de distribuição de energia elétrica? Para responder a pergunta da pesquisa, o objetivo do trabalho foi investigar as informações sociais, ambientais e econômicas que são divulgadas pelos grupos brasileiros de distribuição de energia elétrica alinhadas ao estudo de Siksnelyte e Stjepcevic (2017), tendo em vista que este estudo apresenta mais informações que precisam constar nos relatórios das empresas de energia. Buscou-se assim, através deste estudo, apresentar como as empresas de energia estão preocupadas com a escassez de recursos gerada por sua atividade. A escolha se justifica pela quantidade de itens que precisam constar nos respectivos relatórios. Neste contexto, cabe também referenciar a resolução 
n 444/2001 da ANEEL que obriga suas concessionárias e permissionárias a publicarem o balanço social (ANEEL, 2001), e, todos os setores são impactados pelos crescentes desafios sociais, no entanto, especialmente o setor de energia que estão cada vez mais estimulados a lidar com as questões sociais e ambientais, com maior impacto no bem-estar público e a estabilidade ambiental (SIKSNELYTE; STJEPCEVIC, 2017).

\section{SETOR DE ENERGIA ELÉTRICA NO BRASIL}

As atividades relativas à geração e comercialização de energia elétrica no Brasil têm história recente, teve seu início caracterizado pelo predomínio de empresas estrangeiras, perdurando até as primeiras décadas do século XX, período que começou o processo de estatização do setor, marcado pela verticalização e monopólio das atividades. No entanto, a conjuntura política e econômica de instabilidade que caracterizou a década de 80 , pôs fim à continuidade desse modelo, promovendo um processo de privatização e desverticalização do setor de energia elétrica, quebrando monopólios e permitindo maior flexibilidade no gerenciamento das atividades (AZEVEDO; CRUZ, 2008).

O processo de privatização e desverticalização foi estabelecido no setor de energia elétrica brasileiro a partir da criação das Leis 8.031 de 1990, 8.987 e 9.074 ambas de 1995, esse modelo foi baseado na criação de um mercado competitivo de energia elétrica. Com a Lei 8.031/90 foi instituído o Programa Nacional de Desestatização (PND) e criado o Fundo Nacional de Desestatização (FND). Porém, as desestatizações tiveram seu início a partir de 1995 com as Leis 8.987 e 9.074, que introduziram alterações ao setor elétrico possibilitando a licitação dos novos empreendimentos de geração, a criação da figura do Produtor Independente de Energia, a determinação do livre acesso aos sistemas de transmissão e distribuição e a liberdade para os grandes consumidores escolherem seus supridores de energia (GOMES, et al., 2002).

No ano de 2004, diretrizes foram estabelecidas para o funcionamento do atual modelo do setor elétrico brasileiro, dando alguns importantes passos no sentido de tornar mais seguro o setor elétrico nacional. Para o Ministério das Minas e Energia (MME) os objetivos da criação do novo modelo, são assegurar a eficiência na operação e prestação do serviço aos Consumidores, garantir o controle das tarifas e criar um ambiente regulatório estável que seja estímulo à concorrência, mostrando-se atrativo ao ingresso de novos investimentos privados no setor e que mantenha orientação para as funções de planejamento setorial de longo, médio e curto prazos. (ENGIEE, 2018)

Atualmente o setor elétrico é estruturado sob um conjunto de entidades do governo, públicas e privadas. As principais entidades do setor elétrico brasileiro e suas atribuições básicas podem ser observadas no quadro 1. 
Quadro 1 - Principais entidades do setor elétrico brasileiro e suas atribuições básicas

\begin{tabular}{|c|c|}
\hline $\begin{array}{l}\text { PRINCIPAISENTIDADES DO SE- } \\
\text { TOR ELÉTRICO BRASILEIRO }\end{array}$ & ATRIBUIÇÕES BÁSICAS \\
\hline $\begin{array}{l}\text { Conselho Nacional de Política Energé- } \\
\text { tica- CNPE }\end{array}$ & $\begin{array}{l}\text { O M M E encarrega-se da formulação, do planejamento e da implementa- } \\
\text { ção de ações do governo federal no âmbito da política energética nacional. } \\
\text { Órgão de assessoramento do Presidente da República para formulação de } \\
\text { políticas nacionais e diretrizes de energia, que visa, dentre outros, o apro- } \\
\text { veitamento racional dos recursos energéticos do país, a revisão periódica } \\
\text { da matriz energética e o estabelecimento de diretrizes para programas } \\
\text { específicos. É órgão interministerial presidido pelo Ministro de Minas e } \\
\text { Energia - M M E. }\end{array}$ \\
\hline $\begin{array}{l}\text { Empresa de Pesquisa Energética - EPE } \\
\text { (Dec. no. 5184/2004) }\end{array}$ & $\begin{array}{l}\text { Empresa pública federal dotada de personalidade jurídica de direito } \\
\text { privado e vinculada ao MME. Tem por finalidade prestar serviços na área } \\
\text { de estudos e pesquisas destinadas a subsidiar o planejamento do setor } \\
\text { energético. Elabora os planos de expansão da geração etransmissão da } \\
\text { energia elétrica. }\end{array}$ \\
\hline $\begin{array}{l}\text { Comitê de M onitoramento do Setor } \\
\text { Elétrico - CM SE }\end{array}$ & $\begin{array}{l}\text { Constituído no âmbito do M M E e sob sua coordenação direta, tem a } \\
\text { função de acompanhar e avaliar permanentemente a continuidade ea } \\
\text { segurança do suprimento eletroenergético em todo o território nacional. }\end{array}$ \\
\hline $\begin{array}{l}\text { Operador Nacional do Sistema Elétrico } \\
\text { - ONS (Lei no. 9648/1998) }\end{array}$ & $\begin{array}{l}\text { Entidade jurídica de direito privado, sem fins lucrativos, sob regulação } \\
\text { e fiscalização da ANEEL, responsável pelas atividades de coordenação e } \\
\text { controle da operação da geração e da transmissão de energia elétrica do } \\
\text { Sistema Interligado Nacional (SIN ). }\end{array}$ \\
\hline $\begin{array}{l}\text { Câmara de Comercial ização de Energia } \\
\text { Elétrica- CCEE (Dec. no. 5177/2004) }\end{array}$ & $\begin{array}{l}\text { Entidade jurídica de direito privado, sem fins lucrativos, sob regulação e } \\
\text { fiscalização da ANEEL, tem a finalidade de viabilizar a comercialização de } \\
\text { energia elétrica no SIN e de administrar os contratos de compra e venda } \\
\text { de energia elétrica, sua contabilização eliquidação. }\end{array}$ \\
\hline $\begin{array}{l}\text { A gência Nacional de Energia Elétrica - } \\
\text { ANEEL (Lei n 0. 9427/1996) }\end{array}$ & $\begin{array}{l}\text { Autarquia sob regime especial, vinculada ao MME, tem a finalidade de } \\
\text { regular e fiscalizar a produção, a transmissão, a distribuição e comercia- } \\
\text { lização de energia elétrica, em conformidade com as políticas e diretrizes } \\
\text { do governo federal. É o órgão responsável pela elaboração, aplicação e } \\
\text { atualização dos Procedimentos de Distribuição (PRODIST). }\end{array}$ \\
\hline
\end{tabular}

Fonte: ANEEL (2017)

Entre as principais entidades do setor elétrico brasileiro a ANEEL é encarregada da realização de atividades regulatórias e de fiscalização do setor. $\mathrm{O}$ planejamento das atividades, operação e contabilização são exercidas por empresas públicas ou empresas sem fins lucrativos de direito privado, exemplo da EPE, ONS e CCEE. As atividades de governo são exercidas pelo CNPE, MME e CMSE. As atividades permitidas e reguladas são exercidas pelos demais agentes do setor: Geradores, Transmissores, Distribuidores e Comercializadores (ENGIEE, 2018). 


\section{RESPONSABILIDADE SOCIOAMBIENTAL DAS EMPRESAS DE ENERGIA ELÉTRICA}

Nas últimas décadas, as empresas participantes do setor de energia elétrica têm, crescentemente, diversificado e multiplicado ações destinadas à prevenção, moderação e compensação de impactos de seus empreendimentos sobre o meio ambiente e a sociedade. O aumento do zelo com o desempenho socioambiental de programas de infraestrutura responde a uma tendência global segundo a qual os agentes econômicos têm sido cada vez mais proativos em lidar com as externalidades negativas consequentes das suas atividades (INSTITUTO ACENDE BRASIL, 2017)

O setor de energia elétrica, assim como outros setores que incorrem em grandes obras de infraestrutura, causa mudanças significativas no ambiente natural. As exigências regulatórias para a implantação e operação aumentam, bem como as pressões da sociedade civil e das comunidades locais, em virtude dos impactos gerados à diversidade biológica e ecossistemas (CAIXA,2016)

O reconhecimento por parte das empresas do impacto causado por sua cadeia produtiva e ciclo de serviços que afetam a comunidade local e o meio ambiente está associado com a responsabilidade socioambiental (ANEEL, 2015).

Para atender as exigências das partes interessadas, fornecendo considerações sobre riscos sociais e ambientais que possam afetar a sobrevivência da empresa, bem como as consequências econômico-financeiros relacionados as empresas vêm publicando o Relatório de Responsabilidade Socioambiental (RSA). O RSA no ambiente de energia elétrica tem por objetivo principal ser uma ferramenta de demonstração das políticas e ações específicas desse setor, que evidencie sua ajuda para o desenvolvimento de uma sociedade ambientalmente sustentável, socialmente justa e economicamente viável, mediante inclusão do conceito de um serviço público socialmente responsável (ANEEL, 2006).

É reconhecido que nos últimos anos tenha ocorrido um rápido progresso na publicação de relatórios de responsabilidade socioambiental das empresas, mas acredita-se que ainda que há um longo caminho a ser trilhado no sentido de que esses relatórios atendam as necessidades de seus usuários, os stakeholders, considerando as características de materialidade, credibilidade, integridade e responsabilidade na construção da informação contábil (MOREIRA, et al, 2016).

As empresas do setor elétrico por apresentarem suas atividades destinadas à prestação serviço público, sob o regime de concessão, devem manter a análise da responsabilidade socioambiental ainda mais estendida, passando pela compreensão de que a prestação desses serviços tem de atender, de forma prioritária, ao interesse público, uma vez que toda concessão ou permissão pressupõe a prestação de um serviço apropriado que agrade as circunstâncias de regularidade, continuidade, eficiência, segurança, atualidade, generalidade, cortesia e modicidade das tarifas (ANEEL, 2015).

\subsection{EVIDÊNCIAS EMPÍRICAS}

O estudo de Siksnelyte e Stjepcevic (2017) teve como objetivo analisar o desenvolvimento da responsabilidade social empresarial no sector da energia. É relatado que as empresas de energia têm enfrentado uma série de preocupações relacionadas com questões ambientais e sociais. As autoras concluem que os pilares da RSE no setor de energia são a combinação de incentivos e exigências. As empresas que operam no setor da energia devem entender os impactos sociais, ambientais e econômicos criados em todas as regiões resultantes da realização de suas atividades. 
Lugoboni et al., (2015) buscaram compreender de que forma as empresas do segmento de energia elétrica, listadas na BM\&FBOVESPA como Nível 1 e Novo Mercado de Governança Corporativa divulgam aos interessados seus Relatórios de Sustentabilidade. Na citada pesquisa, realizou-se análise documental, através dos Relatórios de Sustentabilidade das empresas do setor elétrico: CEEE, Cemig, Cesp, Copel, CPFL, CTEEPO, EDP, Eletrobrás, Equatorial, Light, Tractebel e MPX, referentes aos anos de 2010, 2011 e 2012. Os autores concluem que houve uma sutil evolução na divulgação dos indicadores da Global Reporting Initiative GRI, possivelmente por serem reconhecidos internacionalmente, o que proporciona às empresas maior confiabilidade e transparência em suas informações.

Preciado-Hoyos (2013) investigou o papel das relações públicas na gestão da responsabilidade social em um grupo de empresas colombianas do setor elétrico. De acordo com os resultados, foi evidenciado que as comunicações profissionais que apoiam programas de responsabilidade social não possuem um conceito relações públicas. Além disso, a autora constatou que nem todos reconhecem o modelo atualmente vigente, que considera a prática das relações públicas como uma busca pela harmonia com o seu ambiente e benefício mútuo com o interesse público.

Maçambanni et al., (2013) objetivaram identificar o nível de informação socioambiental fornecido pelas empresas de energia do setor elétrico listadas na B3 a partir dos indicadores de responsabilidade social e ambiental proposto pelo modelo Ibase. Os autores buscaram verificar o nível de disclosure dos indicadores sociais e ambientais, por meio do gráfico de barras agrupado, em que observaram que apenas 21 empresas apresentaram um nível de disclosure socioambiental próximo ao exigido. Além disso, a publicação dos indicadores sociais e ambientais mostrou um nível de evidenciação ainda muito aquém do esperado. Dada a resolução da ANEEL que obriga suas concessionárias e permissionárias a publicarem o balanço social, há uma preocupação com o nível de informação socioambiental ainda não totalmente satisfatório.

Gonzaga et al., (2012) avaliaram se o balanço social, divulgado no relatório anual, registrava a responsabilidade social das empresas brasileira de capital aberto. Os indicadores de responsabilidade social apontaram 47,3\% dos indicadores recomendados pela resolução específica do CFC. O balanço social relatado no relatório anual das empresas evidenciava parcialmente a responsabilidade social das empresas brasileiras de capital aberto, sendo a melhor ferramenta de evidenciação de responsabilidade social empresarial à época. Entretanto, na prática, o balanço social ficava prejudicado pela falta de legislação que obrigasse as empresas a usá-lo.

Siqueira e Fernandes (2010) analisaram a qualidade dos balanços sociais de organizações, por meio de quatro parâmetros: abrangência, viés para potencialização dos resultados, comparabilidade e transparência. $\mathrm{O}$ desfecho identificado nesta pesquisa corrobora os diagnósticos anteriormente observados em outros trabalhos e reforça a urgência por mudanças para que sirvam de mensuração de responsabilidade social.

Pelos resultados apresentados por Siksnelyte e Stjepcevic (2017), Lugoboni et al., (2015), Preciado-Hoyos (2013), Maçambanni et al., (2013), Gonzaga et al., (2012) e Siqueira e Fernandes (2010) em seus estudos, pode-se observar que, no quesito da divulgação de informações socioambientais, as empresas de energia elétrica apesar de possuírem uma obrigação na divulgação desse tipo informação, não chegam a atender um nível satisfatório de divulgação. Essa pesquisa trata da mesma perspectiva, no entanto, busca acrescentar informações adicionais neste cenário, ao apresentar os resultados das empresas de energia em relação a exigências mais rígidas apontadas na pesquisa de Siksnelyte e Stjepcevic (2017). 


\section{METODOLOGIA}

Em relação aos procedimentos metodológicos, o presente artigo possui uma abordagem qualitativa, já que para sua realização foram levantadas informações a partir de artigos, relatórios e outros seguidos da interpretação dessas informações. Segundo Gil (2008) a análise qualitativa consiste na organização das informações selecionados de forma a possibilitar a análise sistemática das semelhanças e diferenças e seu inter-relacionamento.

Por ter seus dados coletados a partir de artigos, relatórios, livros e outros esta pesquisa enquadra-se como sendo documental. Como explanado por Marconi e Lakatos (2010), a característica da pesquisa documental é que a fonte de coleta de dados está restrita a documentos, escritos ou não, constituindo o que se denomina de fontes primárias.

Como etapa para o desenvolvimento e conclusão deste artigo foi necessário a análise dos dados coletados, em que foi utilizado o método da análise de conteúdo. A análise de conteúdo pode ser compreendida como um conjunto de técnicas de pesquisa, cujo objetivo é a busca do sentido ou dos sentidos de um documento (CAMPOS, 2004).

O universo da pesquisa foi composto pelos grupos das grandes distribuidoras brasileiras de energia elétrica elencadas no ranking retificado do Desempenho Global de Continuidade do ano de 2017 da ANEEL. A lista com os grupos participantes do universo da pesquisa pode ser observada no quadro 2.

Quadro 2 - Grupos das distribuidoras brasileiras de energia elétrica elencadas no ranking retificado do Desempenho Global de Continuidade do ano de 2017.

\begin{tabular}{|c|c|c|}
\hline DISTRIBUIDORA & GRUPOS & $\begin{array}{c}\text { POSIÇÃO NO } \\
\text { RANKING }\end{array}$ \\
\hline Ceb Distribuição S.A & CEB & 20 \\
\hline Companhia Estadual de Distribuição de Energia Elétrica & CEED & 29 \\
\hline Celesc Distribuição S.A. & CELESC & 21 \\
\hline Cemig Distribuição S.A & CEMIG & 17 \\
\hline Copel Distribuição S.A & COPEL & 19 \\
\hline Companhia Paulista de Força e Luz & \multirow{4}{*}{ CPFL Energia } & 15 \\
\hline Companhia Piratininga de Força e Luz & & 18 \\
\hline Rio Grande Energia S.A. & & 23 \\
\hline Rge Sul Distribuidora de Energia S.A. & & 25 \\
\hline Espírito Santo Centrais Elétricas S.A. & \multirow{2}{*}{ EDP Brasil } & 8 \\
\hline Bandeirante Energia S.A. & & 12 \\
\hline Amazonas Distribuidora de Energia S/A & \multirow{4}{*}{ ELETROBRAS } & 4 \\
\hline Companhia de Energia Do Piauí & & 27 \\
\hline Companhia Energética de Alagoas & & 32 \\
\hline Companhia de Energia de Roraima & & 30 \\
\hline Eletropaulo Metropolitana Eletricidade de São Paulo S.A & ELETROPAULO & 28 \\
\hline Companhia Energética do Ceará & \multirow{3}{*}{ ENEL } & 4 \\
\hline Ampla Energia e Serviços S.A & & 31 \\
\hline Celg Distribuição S.A. & & 33 \\
\hline Energisa Minas Gerais & \multirow{5}{*}{ ENERGISA } & 1 \\
\hline Energisa Sul-Sudeste - Distribuidora de Energia S.A. & & 3 \\
\hline Energisa Paraíba - Distribuidora de Energia & & 4 \\
\hline Energisa Minas Gerais - Distribuidora de Energia S.A & & 10 \\
\hline Energisa Mato Grosso do Sul - Distribuidora de Energia S.A & & 12 \\
\hline
\end{tabular}




\begin{tabular}{|c|c|c|}
\hline Energisa Tocantins - Distribuidora de Energia S.A. & & 12 \\
\hline Energisa Sergipe - Distribuidora de Energia S.A. & & 15 \\
\hline Companhia Energética do Maranhão & \multirow{2}{*}{$\begin{array}{c}\text { EQUATORIAL } \\
\text { ENERGIA }\end{array}$} & 2 \\
\hline Centrais Eletricas do Para & & 9 \\
\hline Light Serviços de Eletricidade S.A. & LIGHT & 22 \\
\hline Elektro Eletricidade e Serviços S.A. & \multirow{4}{*}{ NEOENERGIA } & 7 \\
\hline Companhia Energética do Rio Grande do Norte & & 11 \\
\hline Companhia Energetica de Pernambuco & & 23 \\
\hline Companhia de Eletricidade do Estado Da Bahia & & 26 \\
\hline
\end{tabular}

Fonte: A daptada ANEEL (2018, p.6)

A lista contém um total de 33 empresas com seu respectivo grupo controlador apresentando distribuidoras de todas as regiões do país e onde fazem parte de um total de 14 grupos controladores. A amostra da pesquisa foi constituída pelos relatórios de sustentabilidade do ano de 2017 divulgados pelos grupos controladores.

Os dados foram coletados a partir dos relatórios de sustentabilidade divulgados nos sites de cada grupo participante da amostra da pesquisa, para busca foram utilizados os termos "Responsabilidade social", "Responsabilidade ambiental", "Responsabilidade econômica" e foram consideradas expressões similares de um mesmo campo semântico. Após a coleta dos dados, foi observado como os quesitos relacionados aos pilares mencionados no estudo de Siksnelyte e Stjepcevic (2017) eram citados de algum modo na amostra da pesquisa, por exemplo, no caso do ponto Redução de poluição e emissões, tendo o grupo conseguido ou não reduzir a emissão de poluentes, esse fato deveria ser divulgado em seu relatório.

Em seguida, após a análise dos dados, foi identificado a situação dos grupos das distribuidoras de energia, se estavam ou não alinhadas aos quesitos dos pilares da sustentabilidade apontados no estudo de Siksnelyte e Stjepcevic (2017). Os pilares e as respectivas informações que precisam constar nos demonstrativos das empresas de energia, segundo o estudo, estão dispostos no quadro 3.

Quadro 3 - Responsabilidade social, ambiental e econômica no setor da energia

\begin{tabular}{|c|c|c|}
\hline RESPONSABILIDADE SOCIAL & $\begin{array}{l}\text { RESPONSABILIDADE } \\
\text { AMBIENTAL }\end{array}$ & $\begin{array}{l}\text { RESPONSABILIDADE } \\
\text { ECONÔMICA }\end{array}$ \\
\hline $\begin{array}{l}\text { De bem-estar, habilidades e } \\
\text { motivação pessoal; }\end{array}$ & Medição de impactos ambientais; & Operações rentáveis; \\
\hline Aberto interação com Stakeholders; & $\begin{array}{l}\text { Sistemas de controle de resíduos e } \\
\text { poluição. }\end{array}$ & Preços justos e bom serviço; \\
\hline $\begin{array}{l}\text { A qualidade do fornecimento de } \\
\text { energia; }\end{array}$ & $\begin{array}{l}\text { Minimização da utilização de } \\
\text { combustíveis fósseis; }\end{array}$ & $\begin{array}{l}\text { Investimento em novas } \\
\text { tecnologias; }\end{array}$ \\
\hline Preço correto para a energia. & Redução de poluição e emissões; & $\begin{array}{l}\text { Confiabilidade do fornecimento de } \\
\text { energia; }\end{array}$ \\
\hline \multirow{2}{*}{$\begin{array}{l}\text { Boas práticas de negócios e } \\
\text { cooperação com Stakeholders, em } \\
\text { rede com outras empresas; }\end{array}$} & Fontes renováveis de desenvolvimento; & \multirow[t]{2}{*}{ Gestão de riscos financeiros. } \\
\hline & $\begin{array}{l}\text { Conscientização e redução do meio } \\
\text { ambiente impactos de produção e } \\
\text { transferência da Energia; }\end{array}$ & \\
\hline
\end{tabular}

Fonte: Siksnelytee Stjepcevic (2017) 


\section{RESULTADOS E DISCUSSÕES}

Após a coleta e análise dos dados foi observado que alguns grupos conseguiam atender certos pontos das responsabilidades, mas deixavam de atender outros, como observado no quadro 4.

Quadro 4 - Pontos das responsabilidades atendidos pelos grupos de distribuição de energia elétrica

\begin{tabular}{|c|c|c|c|c|c|c|c|c|c|c|c|c|c|c|c|}
\hline \multicolumn{2}{|c|}{ PONTOS } & 承 & $\frac{2}{2}$ & $\begin{array}{l}0 \\
0 \\
\frac{1}{12} \\
\frac{12}{0} \\
0\end{array}$ & 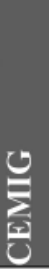 & $\frac{1}{8}$ & $\frac{1}{2}$ & 言 & 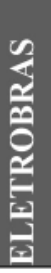 & 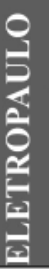 & 졸 & 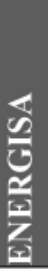 & 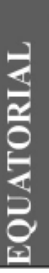 & $\underline{\underline{0}}$ & $\begin{array}{l}\frac{4}{0} \\
\frac{\pi}{2} \\
\frac{1}{2} \\
\frac{x}{2} \\
\frac{1}{2}\end{array}$ \\
\hline \multirow{5}{*}{ 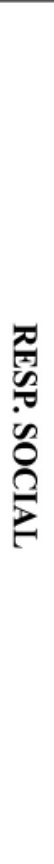 } & $\begin{array}{l}\text { De bem-estar, } \\
\text { habilidades e } \\
\text { motivação pessoal; }\end{array}$ & $x$ & $\mathrm{x}$ & $x$ & $X$ & $\mathrm{x}$ & $\mathrm{x}$ & $x$ & $x$ & $x$ & $X$ & $\mathrm{x}$ & & & $\mathrm{x}$ \\
\hline & $\begin{array}{l}\text { Aberto interação com } \\
\text { Stakeholders; }\end{array}$ & $x$ & $\mathrm{x}$ & $x$ & $X$ & $\mathrm{x}$ & & $x$ & $x$ & $x$ & $X$ & & $x$ & $x$ & $\mathrm{x}$ \\
\hline & $\begin{array}{l}\text { A qualidade do } \\
\text { fornecimento de } \\
\text { energia; }\end{array}$ & $\mathrm{x}$ & $\mathrm{X}$ & & $X$ & $\mathrm{x}$ & & & $x$ & $\mathrm{X}$ & $X$ & $\mathrm{x}$ & $x$ & $X$ & $\mathrm{x}$ \\
\hline & $\begin{array}{l}\text { Boas práticas de } \\
\text { negócios e cooperação } \\
\text { com Stakeholders, em } \\
\text { rede com outras } \\
\text { empresas; }\end{array}$ & $\mathrm{x}$ & $\mathrm{X}$ & $x$ & $X$ & $\mathrm{x}$ & $\mathrm{X}$ & $x$ & $\mathrm{X}$ & $x$ & $X$ & & $x$ & $X$ & \\
\hline & $\begin{array}{l}\text { Preço correto para a } \\
\text { energia. }\end{array}$ & $\mathrm{x}$ & & & $X$ & & $\mathrm{x}$ & & & & & & $x$ & & $\mathrm{x}$ \\
\hline \multirow{6}{*}{ 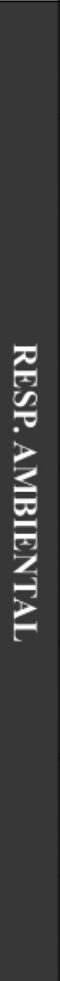 } & $\begin{array}{l}\text { Medição de impactos } \\
\text { ambientais; }\end{array}$ & $x$ & $X$ & $\mathrm{X}$ & $X$ & & $\mathrm{X}$ & $x$ & & $\mathrm{X}$ & $X$ & & & $\mathrm{x}$ & $\mathrm{X}$ \\
\hline & $\begin{array}{l}\text { Conscientização e } \\
\text { redução do meio } \\
\text { ambiente impactos de } \\
\text { produção e } \\
\text { transferência da } \\
\text { Energia; }\end{array}$ & $x$ & & $x$ & $X$ & & $\mathrm{x}$ & $x$ & $x$ & $x$ & $X$ & & & $\mathrm{X}$ & $\mathrm{X}$ \\
\hline & $\begin{array}{l}\text { Minimização da } \\
\text { utilização de } \\
\text { combustíveis fósseis; }\end{array}$ & $\mathrm{x}$ & & & $X$ & $\mathrm{x}$ & $\mathrm{x}$ & $\mathrm{x}$ & & $x$ & $X$ & $\mathrm{x}$ & $x$ & & \\
\hline & $\begin{array}{l}\text { Redução de poluição e } \\
\text { emissões; }\end{array}$ & $x$ & $\mathrm{x}$ & $\mathrm{x}$ & $X$ & $\mathrm{x}$ & $\mathrm{x}$ & $x$ & $x$ & $x$ & $X$ & $\mathrm{x}$ & $x$ & $X$ & \\
\hline & $\begin{array}{l}\text { Fontes renováveis de } \\
\text { desenvolvimento; }\end{array}$ & $x$ & & $\mathrm{x}$ & $X$ & $\mathrm{X}$ & $\mathrm{X}$ & $\mathrm{x}$ & $x$ & $x$ & $X$ & & & & $\mathrm{x}$ \\
\hline & $\begin{array}{l}\text { Sistemas de controle } \\
\text { de resíduos e poluição. }\end{array}$ & $\mathrm{x}$ & & $\mathrm{x}$ & $X$ & $\mathrm{x}$ & $\mathrm{x}$ & $\mathrm{x}$ & $\mathrm{x}$ & $\mathrm{x}$ & $X$ & $\mathrm{x}$ & $x$ & $X$ & $\mathrm{x}$ \\
\hline
\end{tabular}


Continuação Quadro 4

\begin{tabular}{|c|c|c|c|c|c|c|c|c|c|c|c|c|c|c|c|}
\hline \multicolumn{2}{|c|}{ PONTOS } & 옥 & 종 & 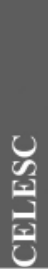 & 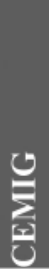 & $\frac{7}{0}$ & $\frac{1}{2}$ & 言 & 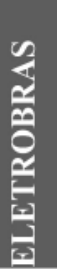 & 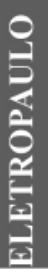 & 国 & $\frac{\sqrt{\frac{\pi}{5}}}{\frac{\pi}{\frac{x}{2}}}$ & 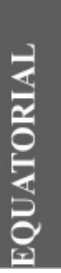 & 息 & 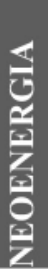 \\
\hline \multirow{5}{*}{ 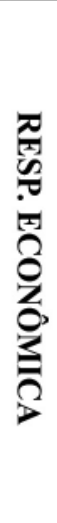 } & Operações rentáveis; & & $\mathrm{x}$ & $\mathrm{x}$ & $\mathrm{X}$ & $\mathrm{x}$ & $\mathrm{x}$ & $\mathrm{x}$ & & & $\mathrm{X}$ & & & & \\
\hline & $\begin{array}{l}\text { Preços justos e bom } \\
\text { serviço; }\end{array}$ & $x$ & $x$ & & $\mathrm{X}$ & $\mathrm{x}$ & $\mathrm{x}$ & & & $\mathrm{x}$ & $\mathrm{X}$ & & $\mathrm{x}$ & & $\mathrm{x}$ \\
\hline & $\begin{array}{l}\text { Investimento em } \\
\text { novas tecnologias; }\end{array}$ & $x$ & $\mathrm{x}$ & $\mathrm{x}$ & $\mathrm{X}$ & $\mathrm{x}$ & $\mathrm{x}$ & $\mathrm{x}$ & $\mathrm{x}$ & $\mathrm{x}$ & $\mathrm{X}$ & $\mathrm{x}$ & $\mathrm{x}$ & $\mathrm{X}$ & $\mathrm{x}$ \\
\hline & $\begin{array}{l}\text { Confiabilidade do } \\
\text { fornecimento de } \\
\text { energia; }\end{array}$ & $x$ & & $\mathrm{x}$ & $\mathrm{X}$ & & & & $\mathrm{x}$ & $\mathrm{x}$ & $\mathrm{X}$ & $\mathrm{x}$ & $\mathrm{x}$ & $\mathrm{X}$ & $\mathrm{x}$ \\
\hline & $\begin{array}{l}\text { Gestão de riscos } \\
\text { financeiros. }\end{array}$ & & & $\mathrm{x}$ & & $x$ & $x$ & $\mathrm{x}$ & & $x$ & $\mathrm{X}$ & & $\mathrm{x}$ & $\mathrm{X}$ & $\mathrm{x}$ \\
\hline
\end{tabular}

Fonte: Dados da pesquisa (2018).

A análise foi feita somente em termos de atendimento ou não nos quesitos relacionados nos pilares mencionados no estudo de Siksnelyte e Stjepcevic (2017), que deveriam constar nos relatórios de sustentabilidade das empresas. Seguindo a análise pode se observar que o atendimento de alguns pontos se sobressaem aos demais, como pode ser visto no quadro 5.

Quadro 5 - Percentual de pontos atendidos pelos grupos das distribuidoras de energia elétrica.

\begin{tabular}{|c|c|c|}
\hline & PONTOS & $\begin{array}{c}\text { PERCENTUAL } \\
\text { GERAL DE PONTOS } \\
\text { ATENDIDOS PELOS } \\
\text { GRUPOS }\end{array}$ \\
\hline \multirow{5}{*}{ 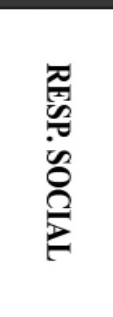 } & De bem-estar, habilidades e motivação pessoal; & $86 \%$ \\
\hline & Aberto interação com Stakeholders; & $86 \%$ \\
\hline & A qualidade do fornecimento de energia; & $79 \%$ \\
\hline & $\begin{array}{l}\text { Boas práticas de negócios e cooperação com } \text { Stakeholders, em rede com } \\
\text { outras empresas; }\end{array}$ & $86 \%$ \\
\hline & Preço correto para a energia. & $36 \%$ \\
\hline \multirow{6}{*}{ 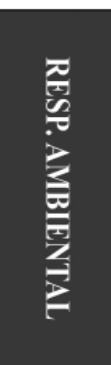 } & Medição de impactos ambientais; & $71 \%$ \\
\hline & $\begin{array}{l}\text { Conscientização e redução do meio ambiente impactos de produção e } \\
\text { transferência da Energia; }\end{array}$ & $71 \%$ \\
\hline & Minimização da utilização de combustíveis fósseis; & $64 \%$ \\
\hline & Redução de poluição e emissões; & $93 \%$ \\
\hline & Fontes renováveis de desenvolvimento; & $71 \%$ \\
\hline & Sistemas de controle de resíduos e poluição. & $93 \%$ \\
\hline \multirow{5}{*}{ 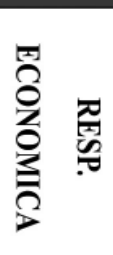 } & Operações rentáveis; & $\mathbf{5 0} \%$ \\
\hline & Preços justos e bom serviço; & $64 \%$ \\
\hline & Investimento em novas tecnologias; & $100 \%$ \\
\hline & Confiabilidade do fornecimento de energia; & $71 \%$ \\
\hline & Gestão de riscos financeiros. & $64 \%$ \\
\hline
\end{tabular}

Fonte: Dados da pesquisa (2018). 
Nesta análise foi verificado quantas e quais empresas atendiam aos pilares da sustentabilidade mencionados no estudo de Siksnelyte e Stjepcevic (2017), com base nessas informações pode ser possível verificar a participação dessas empresas no percentual total da amostra analisada.

No critério Responsabilidade Social o percentual das empresas que abordaram subitens relacionados a esse tema fora acima de $80 \%$ aproximadamente, porém no subitem Preço Correto para energia apenas $36 \%$ das empresas analisadas abordaram esse tema nos seus relatórios.

A grande maioria das empresas apresentaram preocupação ao ponto de bem-estar, habilidade e motivação pessoal. São visíveis em seus relatórios que as empresas procuram profissionais com capacidade, e aperfeiçoam seus colaboradores anualmente, para atuarem com maior motivação em suas atividades. Em praticamente todas as empresas foi possível identificar um aumento no número de funcionárias mulheres, tanto em cargos operacionais quanto em cargos de gestão.

No critério Responsabilidade Ambiental foram identificadas que no mínimo 64\% das empresas abordaram tópicos relacionados ao meio ambiente em seus relatórios. Este fato pode evidenciar que grande parte das empresas de energia elétricas estão preocupadas com os impactos ambientais de suas atividades operacionais. Este fato pode ser também consequência do aumento de exigências em relação a mitigação de impactos ambientais por parte dos seus investidores.

No tocante as Responsabilidades Econômica boa parte das empresas apresentam operações rentáveis em meio à crise econômica que abala o Brasil. Cerca de 100\% das empresas analisadas apresentaram investimentos em grande escala em novas tecnologias para fornecer cada vez mais uma energia limpa, renovável e a um baixo custo. Cerca de $64 \%$ das empresas apresentaram mensuração de indicadores de qualidade do fornecimento de energia, neste quesito pode ser possível identificar que ocorre uma diminuição gradativamente nos indicadores de interrupções no fornecimento de energia.

Ainda seguindo com análise dos resultados pode-se observar o nível de atendimento da responsabilidade social, ambiental e econômica por grupo, conforme o quadro 6.

Quadro 6 - Responsabilidade atendidas pelos grupos das distribuidoras de energia elétrica.

\begin{tabular}{|c|c|c|c|c|}
\hline GRUPO & RESP. SOCIAL & RESP. AMBIENTAL & RESP.ECONOMICA & $\begin{array}{c}\text { PERCENTUAL } \\
\text { GERAL }\end{array}$ \\
\hline CEMIG & $100 \%$ & $100 \%$ & $80 \%$ & $93 \%$ \\
\hline ENEL & $80 \%$ & $100 \%$ & $100 \%$ & $93 \%$ \\
\hline CEB & $100 \%$ & $100 \%$ & $60 \%$ & $87 \%$ \\
\hline ELETROPAULO & $\mathbf{8 0} \%$ & $100 \%$ & $80 \%$ & $87 \%$ \\
\hline CPFL & $60 \%$ & $100 \%$ & $80 \%$ & $80 \%$ \\
\hline COPEL & $80 \%$ & $67 \%$ & $80 \%$ & $76 \%$ \\
\hline NEOENERGIA & $80 \%$ & $67 \%$ & $80 \%$ & $76 \%$ \\
\hline CELESC & $60 \%$ & $83 \%$ & $80 \%$ & $74 \%$ \\
\hline EDP & $60 \%$ & $100 \%$ & $60 \%$ & $73 \%$ \\
\hline EQUATORIAL & $80 \%$ & $50 \%$ & $80 \%$ & $70 \%$ \\
\hline ELETROBRAS & $80 \%$ & $67 \%$ & $40 \%$ & $62 \%$ \\
\hline LIGHT & $60 \%$ & $67 \%$ & $60 \%$ & $62 \%$ \\
\hline CEED & $80 \%$ & $33 \%$ & $60 \%$ & $58 \%$ \\
\hline ENERGISA & $40 \%$ & $50 \%$ & $40 \%$ & $43 \%$ \\
\hline MÉDIA & $74 \%$ & $77 \%$ & $70 \%$ & $74 \%$ \\
\hline
\end{tabular}

Fonte: Dados da pesquisa (2018). 
Em relação aos critérios da Responsabilidade Social pode-se observar que os grupos da CEB e CEMIG atenderam 100\% dos quesitos, enquanto a ENERGISA se apresentou atendendo apenas 40\%, a menor margem do Quadro, o mesmo valor de $40 \%$ foi indicado pela ELETROBRAS na Responsabilidade Econômica. A Responsabilidade Ambiental teve o maior percentual de quesitos atendidos, dentre os 14 grupos 6 conseguiram atender $100 \%$ das questões apontadas no estudo. Analisando o total de quesitos atendidos pelos grupos destacam-se a CEMIG e a ENEM ambas com uma taxa 93\% e não muito distante com $87 \%$ a CEB e a ELETROPAULO.

\section{CONCLUSÕES E RECOMENDAÇÕES}

Esta pesquisa teve como objetivo investigar as informações sociais, ambientais e econômicas que são divulgadas pelos grupos brasileiros de distribuição de energia elétrica. Os principais resultados do presente estudo demonstram que: das catorze empresas analisadas, apenas duas apresentaram percentual abaixo dos $60 \%$ dos itens abordados, numa forma de constatar que elas estão cientes da importância da divulgação de tais informações.

Também foi possível constatar que nos temas relacionados a Responsabilidade Ambiental as empresas apresentaram uma média geral de $77 \%$ de frequência nos temas relacionados a esse tópico em relação ao estudo de Siksnelyte and Stjepcevic (2017) que serviu de base para pesquisa, evidenciando a necessidade de avanço.

Nos outros temas os resultados foram semelhantes, Responsabilidade Social apresentou uma frequência de $74 \%$ enquanto os temas sobre Responsabilidade Economia apresentaram uma frequência de $70 \%$. Desta forma é possível analisar que as empresas apresentaram uma média mínima de 70\% de frequência nos temas abordados por essa pesquisa. Porém, cerca de $36 \%$ das empresas da amostra abordaram o tema de preço correto da energia, o que pode demonstrar que em sua maioria as empresas não estão preocupadas com a cobrança de um preço justo que atenda às necessidades tanto da população quanto dos investidores.

Assim, pôde-se concluir que as empresas estão gerando informações socioambiental, no entanto, assim como os estudos de Gonzaga et al., (2012) e Siqueira e Fernandes (2010), foi constatado a necessidade de melhorias nas ações realizadas pelas empresas de energia, tendo em vista o impacto que a atividade causa na natureza. $O$ estudo contribui ao apresentar lacunas nos pilares sociais, ambientais e econômicos das empresas de energia, gerando a necessidade de ampliação de pesquisas relacionadas ao tema.

O estudo ficou limitado as empresas de energia, especificamente associadas aos grupos de grandes distribuidoras de energia, tendo em vista que o foco principal foi analisar as informações socioambientais e econômicas desse segmento em relação ao estudo de Siksnelyte and Stjepcevic (2017). Assim, sugere-se para pesquisas futuras, a possibilidade de análise sobre toda a cadeia de valor que compreende a geração, transmissão e distribuição de energia. Além disso, outros estudos podem realizar pesquisas comparativas no que se refere aos métodos de evidenciação das responsabilidades ambientais. 


\section{REFERÊNCIAS}

AGÊNCIA NACIONAL DE ENERGIAL ELÉTRICA (ANEEL). Manual de Elaboração do Relatório Anual de Responsabilidade Socioambiental das Empresas de Energia Elétrica.

Disponível em: http://www2.aneel.gov.br/aplicacoes/leitura_arquivo/default.cfm?idaplicacao=43 . Acesso em: 18 abr. 2018.

AGÊNCIA NACIONAL DE ENERGIAL ELÉTRICA (ANEEL). Nota Técnica n⿳0 0044/2018SRD/ANEEL: Retificação do Indicador de Desempenho Global de Continuidade de 2017 (Retificação do resultado apresentado na Nota Técnica $n^{\circ}$ 0027/2018-SRD/ANEEL). Processo $n^{\circ}$ : 48500.000623/2018-13. abr. 2018.

AGÊNCIA NACIONAL DE ENERGIAL ELÉTRICA (ANEEL). Procedimentos de Distribuição de Energia Elétrica no Sistema Elétrico Nacional - PRODIST: Módulo 1 - Introdução. Resolução Normativa nº 345/2008. Brasília/DF, 2008.

AGÊNCIA NACIONAL DE ENERGIAL ELÉTRICA (ANEEL). Resolução nº. 444, de 26 de outubro de 2001. Disponível em: http://www2.aneel.gov.br/aplicacoes/leitura_arquivo/arquivos/ Resolucao444.pdf. Acesso em 18 dez. 2019.

AGÊNCIA NACIONAL DE ENERGIAL ELÉTRICA (ANEEL). Sustentabilidade. Disponível em: http://www.aneel.gov.br/sustentabilidade. Acesso em 11 set. 2018.

ASSOCIAÇÃO BRASILEIRA DE NORMAS TÉCNICAS - ABNT. NBR ISO 26000: diretrizes sobre responsabilidade social. Rio de Janeiro, 2010.

AZEVEDO, T. C.; CRUZ, C. F. Balanço Social como instrumento para demonstrar a responsabilidade social das entidades: Uma discussão quanto à elaboração, padronização e regulamentação. Pensar contábil, v. 8, p. 13-20, 2006.

AZEVEDO, T. C.; CRUZ, C. F. Evidenciação das informações de natureza socioambiental divulgadas pelas distribuidoras de energia elétrica que atuam na região nordeste do brasil e sua relação com indicadores de desempenho empresarial. Enfoque, v. 27, p. 53-66, 2008.

BRAGA, C.; FERREIRA, V. C. Os impactos ambientais no setor de energia elétrica brasileiro e a sua relação com o resultado líquido do exercício. IX Congresso ANPCONT, Curitiba, 2015. CAIXA, Boas Práticas Socioambientais Setor de Energia Elétrica, 2016. Disponível em: http://www.caixa.gov.br/Downloads/Guias-Socioambientais-Caixa/Guia_de_Boas_Praticas_ Socioambientais_Energia.pdf. Acesso em: 11 set. 2018.

CAMILLO, R.; ELY, B. J.; MEDEIROS, F. S. B.; BORTOLUZZI, D. A.; WAECHTER, L. S.; OBREGON, S. Revista de Administração da UFSM, v. 8, ed. Especial, p. 119-132, 2015.

CAMPOS, C. J. G. Método de Análise de Conteúdo: ferramenta para a análise de dados qualitativos no campo da saúde. Rev Bras Enferm, Brasilia, p. 611-614, set./out. 2004.

CONFEDERAÇÃO NACIONAL DA INDÚSTRIA. A Evolução do setor Elétrico Brasileiro Rumo à Sustentabilidade. Disponível em: https://bucket-gw-cni-static-cms-si.s3.amazonaws.com/media/ filer_public/09/de/09de36cb-4f51-4da3-82be-cb9f292269b0/fmase.pdf. Acesso em: 11 set. 2018. ENGIE. Estrutura Institucional do Setor Elétrico. Disponível em: http://www.engieenergia.com. br/wps/portal/internet/negocios/conheca-o-mercado-de-energia/estrutura-institucional-do-setoreletrico. Acesso em: 29 out. 2018.

FINKLER, A.; FINKLER, D. R.; CASTRO, J. L. S.; MILKE, T. F. Relação do Crescimento

Econômico e Consumo de Energia Elétrica. XXIV Seminário de Iniciação Científica da Unijuí. 2016. 
FONTES, R. O informativo definitivo da energia lima: Tudo o que você precisa conhecer de forma simples e direta. Disponível em: https://blog.bluesol.com.br/energia-limpa-conceitos/. Acesso em 18 dez. 2019.

GIL, A.C. Métodos e Técnicas de Pesquisa Social. 6ª Ed. São Paulo: Atlas, 2008. GODÓI DE SOUZA, E.; PEDREIRA, L. S. D.; CAMPOS, H. H.; \& MATTERN, D. A Qualidade dos Relatórios de Sustentabilidade: uma Avaliação em Empresas do Ramo de Energia Elétrica. In: ENCONTRO ANPAD, XXXIII, 2009, Rio de Janeiro. Anais... ENANPAD. Rio de Janeiro: 2009. CD-ROM.

GOMES, A.C. S.; ABARCA, C. D. G.; FARIA, E. A. S. T., FERNANDES, H. H. O. O setor elétrico. In: SÃO PAULO, Elizabeth Maria de; KALACHE FILHO, Jorge. (Org.). BNDES 50 Anos: histórias setoriais. São Paulo: DBA Artes Gráficas, 2002, p. 321-348.

GOMES, J. P. P.; VIEIRA, M. M. F. O campo da energia elétrica no Brasil de 1880 a 2002. Revista de Administração Pública, v. 43, n. 2, p. 295-321, 2009.

GONZAGA, F. M.; LIMA, A. S.; REBELO, L. M. B.; SOUZA, W. A. R. O balanço social e a responsabilidade social nas empresas brasileiras: avaliação em empresas de capital aberto. In: Anais... do IX Convibra Administração-Congresso Virtual Brasileiro de Administração. 2012. GUBIANI, C. A.; SANTOS, V.; BEUREN, I. M. Disclosure ambiental das empresas de energia elétrica listadas no Índice de Sustentabilidade Empresarial (ISE). Sociedade, Contabilidade e Gestão (UFRJ), v. 2, p. 7-23, 2012.

IDEC5 ENERGIA. Energia: A ordem é economizar. Disponível em: http://www.mma.gov.br/ estruturas/secex_consumo/_arquivos/7\%20-\%20mcs_energia.pdf. Acesso em: 31 out. 2018 INSTITUTO ACENDE BRASIL (2017). Custos socioambientais: tendências, ferramentas e responsabilidades. White Paper 19, São Paulo, 28 p. Disponível em: http://www.acendebrasil.com. br/media/estudos/2017_WhitePaper_19_CustosSocioambientais.pdf. Acesso em: 29 out. 2018. LABUSCHAGNE, C.; BRENT, A. C.; VAN ERCK, R. P. Assessing the sustainability performances of industries. Journal of cleaner production, v. 13, n. 4, p. 373-385, 2005.

LICCIARDI, N.; PATRICIO, P.; GOMES, J. K.; BISERRA, S.; SANTOS, V.F. responsabilidade Social Corporativa: Percepções de funcionários de uma empresa recicladora de lâmpadas fluorescentes. South American Development Society Journal, [S.1.], v. 3, n. 9, p. 63, nov. 2017. ISSN 2446-5763.

LOZANO, R.; REID, A. Socially responsible or reprehensible? Investors, electricity utility companies, and transformative change in Europe. Energy Research \& Social Science, v. 37, p. 3743, 2018.

LUGOBONI, L. F.; DE TOLEDO, A. P.; ZITTEI, M. V. M.; SILVA, R. P. Importância da sustentabilidade para as empresas do setor de energia elétrica: utilização de relatório de sustentabilidade com base no GRI. Revista Metropolitana de Sustentabilidade (ISSN 2318-3233), v. 5, n. 3, p. 04-25, 2015.

MAÇAMBANNI, T. A.; VAN BELLEN, H. M.; SILVA, T. L.; VENTURA, C. Evidenciação Socioambiental: Uma Análise do Balanço Social de Empresas do Setor Elétrico que Atuam nas Regiões Sul e Nordeste do Brasil. Revista de Gestão Ambiental e Sustentabilidade-GeAS, v. 2, n. 1, p. 123-142, 2013.

MARCONI, M.A; LAKATOS, E.M. Fundamentos de metodologia científica. 7. ed. São Paulo: Atlas, 2010. 
MOREIRA, F. N.; FERREIRA, A. C. S.; SIQUEIRA, J. R. M.; YRIGARAY, H. A. R. Sombras e Silêncio: Análise dos Relatórios Socioambientais do Setor Elétrico. Sociedade, Contabilidade e Gestão, Rio de Janeiro, v. 11, n. 1, p. 46-69, jan./abr. 2016.

PRECIADO-HOYOS, A. The role of public relations in corporate social responsibility programs in the Colombian electricity sector. Public Relations Review, v. 39, n. 5, p. 591-593, 2013. RODANOV, C. C.; FREITAS, E. C. Metodologia do trabalho científico: métodos e técnicas de pesquisa e do trabalho acadêmico. 2. ed. Novo Hamburgo/RS: Feevale, 2013.

SIDHOUM, A. A.; SERRA, T. Corporate social responsibility and dimensions of performance: An application to US electric utilities. Utilities Policy, v. 48, p. 1-11, 2017.

SILVEIRA, M. L. G.; PFITSCHER, E. D. Responsabilidade Socioambiental: estudo comparativo entre empresas de energia elétrica da Região Sul do Brasil. Perspectivas em Gestão \& Conhecimento, v. 3, n. 2, p. 177-195, 2013.

SIQUEIRA, J.R.M.; FERNANDES, F.S. Balanços Sociais no Brasil: uma análise crítica das práticas corporativas. Revista de Contabilidade do Mestrado em Ciências Contábeis da UERJ, v. 14, n. 2, p. 18-31, 2010.

STJEPCEVIC, J.; SIKSNELYTE, I. Corporate Social Responsibility in Energy

Sector. Transformations in Business \& Economics, v. 16, n. 1, 2017.

UNIÃO EUROPEIA. Ambiente. Disponível em:https://europa.eu/european-union/topics/ environment_pt. Acesso em: 30 nov. 2018. 